\title{
Assistência odontológica ao paciente diabético
}

\author{
Dental care to the diabetic patient
}

Atención dental al paciente diabético

José Milton de Aquino e Silva Neto ${ }^{1 *}$, Lara Beatriz Henrique Lima1, Glayce Kelly Siqueira Gomes ${ }^{1}$, Izabel Cristina Gomes de Mendonça'.

\section{RESUMO}

Objetivo: Analisar por meio de uma revisão narrativa sobre os cuidados odontológicos no atendimento aos pacientes diabéticos. Revisão bibliográfica: $O$ diabetes é um distúrbio metabólico heterogêneo, de etiologia múltipla, caracterizado pelo aumento dos níveis glicêmicos no sangue resultante da produção insuficiente de insulina pelo pâncreas, por se tratar de uma doença crônica e irreversível, caracterizada por uma infinidade de complicações locais e sistêmicas como hipoglicemia, hiperglicemia, aterosclerose, retinopatia, nefropatia, neuropatias, úlceras diabéticas e o aumento da suscetibilidade a infecções. Com isso, evidências correlacionadas a odontologia, ressaltam que existe uma interligação entre pacientes diabéticos e o desenvolvimento de periodontopatias assim como implicações imunológias e alterações nas citocinas próinflamatórias, as quais afetam significativamente o processo de cicatrização de feridas cirúrgicas e a saúde dos tecidos da cavidade oral. Considerações finais: Nesse cenário, é de crucial importância o conhecimento do diabetes e suas complicações orais e sistêmicas, pelos Cirurgiões Dentistas (CD), antes de iniciar qualquer protocolo de tratamento em pacientes portadores dessa patologia, para que possa se cercar dos cuidados necessários a fim de evitar problemas que possam afetar permanentemente a vida dessas pessoas.

Palavras-chave: Assistência odontológica, Diabetes mellitus, Assistência integral à saúde.

\begin{abstract}
Objective: Analyze through a narrative review on dental care in the care of diabetic patients. Literature review: Diabetes is a heterogeneous metabolic disorder, of multiple etiology, characterized by an increase in glycemic levels in the blood resulting from insufficient production of insulin by the pancreas, as it is a chronic and irreversible disease, characterized by an infinity of local and systemic complications such as hypoglycemia, hyperglycemia, atherosclerosis, retinopathy, nephropathy, neuropathies, diabetic ulcers and increased susceptibility to infections. Thus, evidence related to dentistry, highlights that there is an interconnection between diabetic patients and the development of periodontopathies as well as immunological implications and changes in pro-inflammatory cytokines, which significantly affect the healing process of surgical wounds and the health of the tissues of the oral cavity. Final considerations: In this scenario, the knowledge of diabetes and its oral and systemic complications by the Dental Surgeons (CD) is of crucial importance before starting any treatment protocol in patients with this pathology, so that they can surround themselves with the necessary care in order to avoid problems that can permanently affect these people's lives.
\end{abstract}

Key words: Dental care, Diabetes mellitus, Comprehensive health care.

\section{RESUMEN}

Objetivo: Analizar a través de una revisión narrativa sobre la atención odontológica en la atención de pacientes diabéticos. Revisión bibliográfica: La diabetes es un trastorno metabólico heterogéneo, de

${ }^{1}$ Centro Universitário CESMAC (CESMAC), Maceió - AL. *E-mail: milton_neto_166@hotmail.com 
etiología múltiple, caracterizado por un aumento de los niveles de glucemia en sangre resultante de la producción insuficiente de insulina por parte del páncreas, por ser una enfermedad crónica e irreversible, caracterizada por una infinidad de complicaciones locales y sistémicas como la hipoglucemia. , hiperglucemia, aterosclerosis, retinopatía, nefropatía, neuropatías, úlceras diabéticas y mayor susceptibilidad a infecciones. Así, la evidencia relacionada con la odontología, destaca que existe una interconexión entre los pacientes diabéticos y el desarrollo de periodontopatías, así como implicaciones inmunológicas y cambios en las citocinas proinflamatorias, que afectan significativamente el proceso de cicatrización de las heridas quirúrgicas y la salud de los tejidos del paciente. Cavidad oral. Consideraciones finales: En este escenario, el conocimiento de la diabetes y sus complicaciones orales y sistémicas por parte de los Cirujanos Dentistas (CD) es de crucial importancia antes de iniciar cualquier protocolo de tratamiento en pacientes con esta patología, para que puedan rodearse de los cuidados necesarios para evitar problemas que pueden afectar de forma permanente la vida de estas personas.

Palabras clave: Atención odontológica, Diabetes mellitus, Atención integral de salud.

\section{INTRODUÇÃO}

A população brasileira vem, ao longo das últimas décadas, passando por transformações que saltam aos olhos, no que se refere ao tempo de vida das pessoas. Na década de 1960 o número de idosos no Brasil que girava em torno de 3 milhões, conta agora com aproximadamente 30 milhões de indivíduos; essa mudança se deu principalmente pelas conquistas alcançadas no âmbito das políticas socioeconômicas e de saúde implementadas graças a influência das diretrizes e orientações internacionais expressas na Constituição Federal promulgada em 1988 (VERAS RP e OLIVEIRA M, 2018). Porém, esse ganho em anos vividos veio acompanhado por morbidades inerentes ao desgaste fisiológico normal ao processo de envelhecimento. $O$ diabetes é um desses problemas, um levantamento feito em 2014 constatou que 422 milhões de pessoas no mundo eram portadoras da doença (MIRANDA GMD, et al., 2016; KANTER JE e BORNFELDT KE, 2016).

O diabetes é um distúrbio metabólico heterogêneo, de etiologia múltipla, caracterizado pelo aumento dos níveis glicêmicos no sangue resultante da produção insuficiente de insulina pelo pâncreas (PAPATHEODOROU K, et al., 2016). Trata-se de uma doença crônica e irreversível, caracterizada por uma infinidade de complicações locais e sistêmicas como hiperglicemia, aterosclerose, retinopatia, nefropatia, neuropatias, úlceras diabéticas, aumento da suscetibilidade a infecções entre as quais estão o pé diabético, catarro, celulite, fasceíte necrosante e infecções do trato urinário (CERVINO G, et al., 2019).

Esta maior susceptibilidade ao desenvolvimento de infecções é preocupante quando o diabético necessita de cuidados odontológicos, dada a natureza contaminada do ambiente oral. Evidências correlacionam pacientes diabéticos e o desenvolvimento de periodontopatias assim como implicações imunológicas e alterações nas citocinas pró-inflamatórias, as quais afetam significativamente o processo de cicatrização de feridas cirúrgicas e a saúde dos tecidos orais (PAPATHEODOROU K, et al., 2016; CERVINO G, et al., 2019).

Os pacientes que apresentam Diabetes Melittus se não possuírem atenção e cuidados periódicos com o Cirurgião Dentista (CD), podem ocorrer inúmeras alterações na cavidade bucal, como são os casos de infecções fúngicas, patologias que envolvem o sistema periodontal, modificações nas glândulas salivares, acarretando diminuição de saliva e cicatrização modificada. Nesse cenário, é de crucial importância o conhecimento do diabetes e suas complicações orais e sistêmicas, pelos Cirurgiões Dentistas, antes de iniciar qualquer protocolo de tratamento em pacientes portadores dessa patologia, para que possa se cercar dos cuidados necessários a fim de evitar problemas que possam afetar permanentemente a vida dessas pessoas (ÁLAMO SM, et al., 2011; UNGER J, 2011).

Portanto, o objetivo desse trabalho é pesquisar na literatura especializada a etiologia, os tipos, a evolução e o tratamento do diabetes, bem como suas complicações e fatores de risco, e proporcionar ao cirurgiãodentista a base de conhecimento necessária para conduzir um tratamento seguro em seus pacientes diabéticos. 


\section{REVISÃO BIBLIOGRÁFICA}

O termo diabetes Mellitus refere-se a um grupo de doenças que compartilham características em comum, nas quais o aumento dos índices de glicose na corrente sanguínea é o fator mais relevante, são, portanto, doenças caracterizadas por uma hiperglicemia causada por vários fatores, dentre eles destacam-se os fatores genéticos, processos inflamatórios, e até mesmo um câncer, ou seja, sua etiologia é multifatorial e ela pode se manifestar em decorrência de outras doenças, como fatores endócrinos, por exemplo, porém, independente do fator etiológico pode-se observar duas linhas fisiológicas dessa doença (CANTANHEDE ALC, et al., 2013).

Em uma dessas linhas fisiológicas ocorre uma resistência periférica à ação da insulina, ou seja, a produção do hormônio é aparentemente normal, mas, por algum motivo os receptores de insulina na célula não permitem uma ação efetiva da mesma acontecendo o que se convencionou chamar de resistência adquirida à ação da insulina. Na outra vertente ela se manifesta por uma deficiência na secreção da insulina que pode ser relativa, quando há produção de insulina, mas a quantidade secretada está aquém da que o organismo necessita para agir corretamente no controle da glicose no sangue, ou absoluta, quando o pâncreas para de produzir insulina, porém, é muito comum casos de pacientes que apresentam os dois tipos (PRESHAW PM, et al., 2012; PETERSMANN A, et al., 2019).

Existem casos em que essas duas linhas atuam de forma conjunta, ou seja, ao mesmo tempo que o paciente tem uma deficiência da secreção de insulina, desenvolve também uma resistência periférica à ação da mesma, por isso a importância da insulina no que se refere a patogenia dos pacientes que apresentam diabetes, quando a quantidade de insulina não apresenta-se de maneira satisfatória, ou seja quando há uma resistência à sua ação, ocorre uma dificuldade na diminuição da concentração de glicose no sangue e principalmente da entrada de glicose para o meio intracelular, visto que é um substrato importante de energia e explica muito das complicações e dos sintomas associados ao diabetes (PEREIRA DR, et al., 2011; SANTOS-PAUL MA, et al., 2015).

O diabetes é classificado em quatro grandes grupos: Diabetes Mellitus tipo I, Diabetes Mellitus tipo II, Diabetes mellitus gestacional e outros tipos específicos que se enquadram como sendo tipos raros de diabetes. Essa classificação em grupos é de suma importância para o direcionamento do profissional na definição da abordagem terapêutica endócrina que deverá seguir (OLIVERA AR, et al., 2017; PETERSMANN A, et al., 2019).

\section{Tipos de Diabetes Mellitus}

Segundo American Diabetes Association (2013), o Diabetes Mellitus pode dispor da respectiva especificação: Diabetes Mellitus dependente de insulina (DMDI), Diabetes em criança e Diabetes Mellitus não dependente de insulina (DMNDI) ou Diabetes do adulto. Buscando evitar diagnósticos sem conformidades, a forma para se classificar foi circunscrita em duas terminologias, o Diabetes tipo I do qual encontra-se o grupo DMDI e Diabetes tipo II para o grupo DMNDI (CARLESSO GP, et al., 2017; GALDINO YLS, et al., 2019).

Entre os usuários que possuem o DM, $10 \%$ apresentam diabetes tipo I, definidos pela forma que as células beta do pâncreas são prejudicadas, em decorrência de processos virais ou patologias que são auto-imunes, promovendo carência na formação de insulina. Na maioria das vezes, o DM tipo I possui sua evolução em pessoas mais jovens, com a faixa etária de idade inferior a 25 anos (GALDINO YLS, et al., 2019; PETERSMANN A, et al., 2019).

Por outro lado, o diabetes tipo II retrata-se como sendo a forma mais encontrada da doença, ocasionada pela atenuação na sensibilidade dos tecidos que são alvos e pela ausência ou improdutividade dos agentes responsáveis por receptar células da insulina excretada pelo pâncreas. Esses aspectos são relacionados a inúmeros fatores, dos quais pode ser destacado: idade do paciente, origem étnica, fatores genéticos, pessoas que são sedentárias e na gestação, essa doença apresenta consequência significativa tanto para a mãe quanto para o bebê, possuindo maior risco de macrossomia fetal, cirurgia de cesariana, risco de hipertensão materna, possibilidade de a mãe desenvolver o diabetes tipo 2 após o parto e a criança ser mais suscetível à obesidade e intolerância à glicose no futuro. Outro fator que pode favorecer o aparecimento da diabetes tipo 
Il é a obesidade prolongada do indivíduo, visto que pode esta correlacionada à insulina (MOREY-VARGAS OL e SMITH AS, 2015; GALDINO YLS, et al., 2019).

Observa-se que atualmente a prevalência do diabetes Mellitus é altíssima no mundo, possuindo 415 milhões de portadores, à uma estimativa de muitos pacientes que não foram diagnosticados e a uma projeção para que a incidência de diabetes aumente cada vez mais, sendo observado em estudos que em 2040 exista 642 milhões de pacientes portadores de diabetes (BARBIERI J, et al., 2015; COSTA RM, et al., 2016).

Nos Estados Unidos observa-se que a prevalência nos dias de hoje são de 9,4\% da população adulta e $24 \%$ não diagnosticados, a prevalência na população idosa que possui idade $\geq$ que 65 anos representa $25 \%$ ou seja, um quarto de cada idoso $\geq$ de 65 anos é portador de diabetes, sendo mais alta ainda essa prevalência de pré diabetes que é um fator de risco importante para o diabetes, no Brasil possui dados da pesquisa nacional dos quais demonstram que a prevalência de diabetes da população é em média de $6 \%$ e ela vem aumentando de acordo com a idade do indivíduo, chegando em aproximadamente $20 \%$ em pessoas com idade acima de 60 anos, um dos fatores que fazem com que haja esse crescimento é o número de pessoas obesas (BARCELÓ A, et al., 2016).

\section{Sintomatologia do Diabetes Mellitus}

Os sintomas clássicos do diabetes são: poliúria que é o aumento do volume urinário, em função da grande concentração de glicose na circulação sanguínea, ocasionando a excreção de uma grande quantidade de glicose e por isso há uma perda de líquido muito grande na urina e consequentemente existira o segundo sintoma que é polidipsia, ou seja, um aumento da sede, perda de peso implicada, o indivíduo mesmo sem mudar o hábito nenhum em termos de alimentação, atividade física, isso porque a glicose é um substrato de energia importante para o meio intracelular, se a glicose não entra na célula, ocasiona uma dificuldade no organismo, fazendo com que o organismo comece a utilizar outras fontes de energia por exemplo a gordura, por isso a perda de peso (NETA R, et al., 2015).

Pode desencadear também a polifagia que acarreta a sensação de fome, a glicose que não é secretada vai se acumulando em determinados órgãos, gerando o que se conhece como espécies reativas de oxigênio, ocasiona também alteração visual que prejudica a retina ocasionando alterações visuais e fraqueza entre os pacientes com diabetes (NETA R, et al., 2015; PETERSMANN A, et al., 2019).

Os Cirurgiões Dentistas não realizam o diagnósticos, porém cabe identificar se há uma alteração ou não nos parâmetros metabólicos de maneira técnica, o diagnóstico é realizado pelo médico, um exame só não é suficiente para um diagnóstico concreto, para isso é necessário fazer outro exame em um período mais curto possível para confirmação do diagnóstico do diabetes (AMERICAN DIABETES ASSOCIATION, 2013). A Associação Americana de Diabetes, determina quatro exames para o resultado se o paciente tem ou não diabete, conforme estabelecido na Tabela 1.

Tabela 1 - Diagnósticos para averiguar se o paciente possui diabetes Mellitus.

\begin{tabular}{|l|c|c|}
\hline Exames & Pré-diabetes & Diabetes \\
\hline Glicose em jejum & $100-125 \mathrm{mg} / \mathrm{dl}$ & $\geq 126 \mathrm{mg} / \mathrm{dl}$ \\
\hline Glicose plasmática $2 \mathrm{~h}(75 \mathrm{~g})$ & $140-199 \mathrm{mg} / \mathrm{dl}$ & $\geq 200 \mathrm{mg} / \mathrm{dl}$ \\
\hline Hemoglobina glicada (controle glicêmico). & $5,7-6,4 \%$ & $\geq 6,5 \%$ \\
\hline $\begin{array}{l}\text { Glicemia ao acaso (quando o paciente apresenta } \\
\text { sintomatologia ou crise de hiperglicemia. }\end{array}$ & & $\geq 200 \mathrm{mg} / \mathrm{dl}$ \\
\hline
\end{tabular}

Fonte: Neto JMAS, et al., 2021. Dados extraídos da American Diabetes Association, 2013.

Então são esses exames, nessas respectivas referências que deve ser utilizado para que o Cirurgião Dentista saiba determinar o grau de controle do paciente e o profissional planeje um tratamento odontológico adequado (COSTA RM, et al., 2016).

$\mathrm{Na}$ área odontológica uma das complicações que tem maiores intercorrências de acordo com a organização mundial de saúde é a doença periodontal, especificamente a periodontite dos quais provocam 
uma Infecção na parte gengival de forma grave que danifica as gengivas e pode destruir o osso maxilar, sendo considerada uma complicação clássica do diabetes melittus (PEREIRA DR, et al., 2011; SANTOS-PAUL MA, et al., 2015).

\section{Pacientes com diabetes na Odontologia}

Visando manter uma melhor restrição do paciente que possui a DM, é de extrema relevância possuir atenção como encontra-se a saúde de maneira geral, as imposições do indivíduo e os recursos presentes, oferecidos para eles. $O$ atendimento adequado e o diagnóstico precoce pode diminuir de maneira significativa os gastos econômicos que venham a comprometer a família. A incorporação de uma equipe multiprofissional visando o progresso através de práticas educativas que buscam como principal proposito aumentar os níveis de saúde é certamente fundamental para uma administração adequada destes pacientes portadores (PAPATHEODOROU K, et al., 2016).

O CD deve ser capaz de tratar o indivíduo diabético sem colocar em risco seu estado de saúde presente, ele pode e deve contribuir para identificar casos que ainda não foram diagnosticados, levando em consideração a taxa de mortalidade presente atualmente de pacientes que não sabem que possuem essa patologia, dessa maneira $O C D$ contribui, buscando reduzir o número de indivíduos que vão para uma hemodiálise por exemplo (PAPATHEODOROU K, et al., 2016; CERVINO G, et al., 2019).

Cabe ao CD prevenir complicações durante o procedimento a ser realizado e possíveis complicações futuras para o paciente, as principais manifestações bucais são: periodontite sendo uma das causas principais, a xerostomia é retratada de 10 à $30 \%$ dos pacientes diabéticos, acredita-se que essa alteração salivar deve-se em função da grande perda de volume na urina em função da excreção de glicose de maneira colúria, então talvez seja decorrente mais ainda não sabe-se qual seria o exato mecanismo que levaria por exemplo ao relato de xerostomia, infecções bacterianas, virais e fúngicas, retardo na cicatrização de feridas orais, em função da alteração salivar cárie também pode ser uma manifestação frequente não de forma direta e sintomas da síndrome da ardência bucal devido a neuropatia (ÁLAMO SM, et al., 2011; SANTOS-PAUL MA, et al., 2015).

As ações de precauções utilizadas em pacientes que apresentam diabetes mellitus são de suma importância, especialmente quando se tem em vista a ampliação dos fatores de risco de patologias periodontais em indivíduos diabéticos (TERRA BG, et al., 2011). Faz-se essencial, assim sendo, abranger nas condutas clínicas um minucioso parecer avaliativo da integridade dos tecidos que envolvem o periodonto, além de ser indicado com bastante frequência profilaxias, sem esquecer de falar sobre a importância da escovação dental diária e a limpeza com fio dental, esses fatores são levados em consideração devido o motivo de que os indivíduos portadores dessas doenças, apresentam maior vulnerabilidade de ter problemas periodontais podendo chegar até mesmo uma reabsorção óssea alveolar, grande chance de inflamações gengivais (PINHO P, 2011; CANTANHEDE ALC, et al., 2013).

O diabetes tipo II sem sombra de dúvida é a mais prevalente, sendo, consequentemente, o tipo de diabéticos mais frequentes no consultório odontológico. Esse tipo da doença é também conhecido como diabetes não insulino dependente, visto que os pacientes apresentam resistência à insulina ou deficiência relativa da mesma. Normalmente o paciente apresenta produção de insulina satisfatória, mas por algum motivo o número de receptores nas células não é suficiente, tendo o organismo uma tendência de aumentar a produção da insulina para compensar essa resistência, então um paciente que apresentava uma produção normal de insulina ao adquirir o diabete, passa a ter uma produção excessiva de insulina e progressivamente ocorre à perda na produção de insulina, a maioria dos casos acomete mais indivíduos obesos, o que torna a obesidade um fator de risco do tipo II, aproximadamente 60 à $70 \%$ dos casos os índices de diabetes tipo II estão correlacionados em indivíduos com a obesidade (MAFFACCIOLLI R e OLIVEIRA DLLC, 2018).

De acordo com Maffacciolli R e Oliveira DLLC (2018), para a realização de uma boa consulta, o paciente portador da DM deve possuir o seu metabolismo de maneira compensada, tendo que estar sendo acompanhado por um bom médico de maneira regular, dispondo de um bom retorno terapêutico. Nos em que o paciente não apresente um quadro de diabetes compensado, na maioria das vezes propendem possuir 
ocorrências indesejáveis no tratamento proposto pelo $C D$ fazendo com quer ocorra dificuldade de estabelecer um procedimentos terapêuticos adequado, podendo surgir infecções futuras devido o processo de cicatrização que é dificultado nesses indivíduos.

Quando ocorre essas intercorrências é imprescindível que o CD faça as medidas paliativas e quando o paciente encontrar compensado continuar o tratamento nas sessões posteriores, porém é necessário a estabilidade do quadro clinico do paciente. Fatores como ansiedade e nervosismo dos pacientes devem serem comedidos, posto que essas sintomatologias levam à liberação de epinefrina e, consequentemente, ao aumento da glicemia (BARCELÓ A, et al., 2016).

\section{Horário das consultas}

A consulta feita pelos Cirurgiões Dentistas aos portadores de diabetes devem ser ajustados de acordo com a particularidade de cada um, tendo-se como atenção os horários e o tempo de procedimentos clínicos realizado em uma sessão (SANTOS-PAUL MA, et al., 2015). Estudos demonstram que o melhor atendimento é realizado no período da manhã, já que a insulina conseguem chegar em seu grau culminante de secreção. De maneira complementar, no decorrer da manhã, os graus endógenos de corticosteroides em que são responsáveis pelo efeitos anti-inflamatórios e imunossupressores encontram-se mais altos, consentindo melhor flexibilidade do paciente portador ao aumento da adrenalina que é um hormônio simpaticomimético e neurotransmissor responsável por preparar o organismo para a realização de grandes feitos, e da glicemia que é a concentração de glicose no sangue ou mais precisamente no plasma, dos quais são resultantes em decorrência de ocasiões de estresse (BARCELÓ A, et al., 2016; CERVINO G, et al., 2019).

Quando a consulta for um pouco demorada, deve-se saber o estado clínico do paciente e explicar ao mesmo os procedimentos que irão serem realizados, visto que esses procedimentos podem acarretar ansiedade nos pacientes. Além do que, cabe ao profissional de saúde falar para o paciente ir alimentado para a consulta que irá ser realizada (CARNEIRO LÚCIO OS e BARRETO RC, 2012). O Cd deve elucidar a respeito da apropriada dieta a ser estabelecida e também quanto a forma correta de ser realizada o processo de higienização da cavidade bucal, assim como aferir a pressão quando o paciente chegar e depois do tratamento realizado (TERRA BG, et al., 2011).

Em casos que o CD começar a fazer o tratamento e o paciente não se sentir bem, apresentando quadros de hipoglicemia é necessário que o profissional pare o procedimento que está sendo executado, tranquilizálo e oferecer algum alimento, com o objetivo de reverter o quadro clínico do paciente, sempre buscando conversar com o mesmo e aferir a pressão arterial (TERRA BG, et al., 2011; PETERSMANN A, et al., 2019).

\section{Periodontite em pacientes com diabete}

O indivíduo que possui essa patologia apresenta uma hiperglicemia em que ocorre a formação e acúmulo de produtos finais da glicosilação avançada (AGE), ocasionando a patogenia do diabetes e danos a órgãos, os AGE se ligam aos monócitos e macrófagos acarretando a resposta hiperinflamatória, devido essa inflamação o paciente terá maior profundidade de sondagem, perda de inserção. Por outro lado esses mesmos produtos de glicose se ligam a células como os neutrófilos que são células importantes de defesa dos quais impedem a invasão e penetração bacteriana porém mesmo assim ocorre a invasão bacteriana. Ou seja, ocorre a diminuição de neutrófilos com isso há uma maior penetração bacteriana, essas bactérias vão ativar as células inflamatórias que também estão com o fenótipo alterado pelo diabetes e vão gerar uma maior produção de citocinas inflamatórias, levando mais informação e acarretando destruição tecidual, com isso vão ter grande perda de inserção a perda óssea, acarretando por sua vez bolsas periodontais que é um ambiente extremamente favorável ao crescimento e colonização de microorganismos fazendo com que esse processo perpetue (PAPATHEODOROU K, et al., 2016; CERVINO G, et al., 2019).

Apresentam uma maior prevalência em indivíduos que não apresenta um controle da doença, o paciente descompensado terá uma pior resposta à terapia periodontal, então quando o CD vai tratar um paciente diabético precisa-se de uma abordagem conjunta com o endocrinologista desse paciente para buscar melhorar o controle glicêmico, visto que essa conduta irá estabelecer um tratamento com resultados benéficos e uma boa cicatrização (PINHO P, 2011; CANTANHEDE ALC, et al., 2013). 


\section{CONSIDERAÇÕES FINAIS}

Sabendo-se que o Diabetes Mellitus é uma patologia das quais envolvem grande parte da saúde pública, dos quais necessita-se de uma maior atenção e cuidados, não simplesmente por parte do $C D$, sendo necessário um acompanhamento bem direcionado por uma equipe multiprofissional. Tais profissionais necessitam possuir uma capacitação, além de buscar sempre estar comprometidos com uma boa prática de maneira preventiva desta patologia. No decorrer do tratamento terapêutico realizado pelo $C D$, deve-se estabelecer primeiramente uma minuciosa anamnese para saber qual conduta irá ser empregue em cada indivíduo objetivando assim um atendimento de maneira particularizada, propiciando serenidade e bem-estar aos pacientes ao longo de todo o tratamento.

\section{REFERÊNCIAS}

1. ÁLAMO SM, et al. Dental considerations for the patient withdiabetes. J ClinExpDent. 2011; 3(1): 25-30.

2. AMERICAN DIABETES ASSOCIATION. Diabetes Management in Correctional Institutions. Diabetes Care. 2013; 36(1): 86-92.

3. BARBIERI J, et al. Anemia in Patients with Type 2 Diabetes Mellitus. Anemia. 2015; 20; 354.

4. BARCELÓ A, et al. Hyperglycemia and pregnancy in the americas. Final Report of the Pan American Conference on Diabetes and Pregnancy. Washington, D.C.; 2016.

5. CANTANHEDE ALC, et al. O idoso portador de diabetes mellitus sob a perspectiva odontológica. Rev Bras Clin Med. São Paulo. 2013; 11(2): 178-82.

6. CARLESSO GP, et al. Evaluation of diabetic patients knowledge about preventive care of the diabetic foot, in Maringá, PR, Brazil. J Vasc Bras. 2017; 16(2): 113-8.

7. CARNEIRO LÚCIO PS, BARRETO RC. Emergências Médicas no Consultório Odontológico e a (In)Segurança dos Profissionais. Rev. bras. ciên. saúde. 2012; 16(2): 267-272.

8. CERVINO G, et al. Diabetes: Oral Health Related Quality of Life and Oral Alterations. Biomed Res Int. 2019.

9. COSTA RM, et al. O Paciente Diabético na Clínica Odontológica: Diretrizes Para o Acolhimento e Atendimento. R bras ci Saúde. 2016; 20(4): 333-340.

10. GALDINO YLS, et al. Validation of a booklet on self-care with the diabetic foot. Rev Bras Enferm. 2019; $72(3): 780-7$.

11. KANTER JE, BORNFELDT KE. Impact of Diabetes Mellitus. Arterioscler Thromb Vasc Biol. 2016; 36(6): $1049-53$.

12. MAFFACCIOLLI R, OLIVEIRA DLLC. Challenges and perspectives of nursing care to vulnerable populations. Rev Gaúcha Enferm. 2018; 39: 217-289.

13. MIRANDA GMD, et al. O envelhecimento populacional brasileiro: desafios e consequências sociais atuais e futuras. Rev. Bras. Geriatr. Gerontol. Rio de Janeiro, 2016; 19(3): 507-519.

14. MOREY-VARGAS OL, SMITH SA. Be Smart: strategies for foot care and prevention of foot complications in patients with diabetes. Prosthet Orthot Int. 2015; 39(1): 48-60.

15. NETA R, et al. Adherence to foot self-care in diabetes mellitus patients. Rev Bras Enferm. 2015; 68(1): 111-6.

16. OLIVERA AR, et al. Comparação de algoritmos de aprendizagem de máquina para construir um modelo preditivo para detecção de diabetes não diagnosticada - ELSA-Brasil: estudo de acurácia. São Paulo Med J. 2017; 135(3): 234-46.

17. PAPATHEODOROU K, et al. Complications of Diabetes. J Diabetes Res, 2016.

18. PEREIRA DR, et al. A influência do tratamento periodontal no controle glicêmico em pacientes diabéticos tipo 2 não insulino-dependentes: artigo original Periodontia 2011; 21(1): 3442.

19. PETERSMANN A, et al. Definition, Classification and Diagnosis of Diabetes Mellitus. Exp Clin Endocrinol Diabetes. $2019 ; 127(1): 1-7$.

20. PINHO P. Diabete Melito: considerações gerais para o cirurgião-dentista. Rev Periodontia 2011; 21(1): $10-1$.

21. PRESHAW PM, et al. Periodontitis and diabetes: a twoway relationship. Diabetologia. 2012; 55(1): 21-31.

22. SANTOS-PAUL MA, et al. Local anesthesia with epinephrine is safe and effective for oral surgery in patients with type 2 diabetes mellitus and coronary disease: a prospective randomized study. Clinics (São Paulo). 2015; 70(3): 185-9.

23. TERRA BG, et al. O cuidado do paciente odontológico portador de diabetes mellitus tipo 1 e 2 na Atenção Primária à Saúde. Rev APS. 2011; 14(2): 149-161.

24. UNGER J. Insulin initiation and intensification in patients with T2DM for the primary care physician. Diabetes Metab Syndr Obes., 2011; 4(1): 235-61.

25. VERAS RP, OLIVEIRA M. Envelhecer no Brasil: a construção de um modelo de cuidado. Ciênc. Saúde. Colet. 2018; 23(6): 201-209. 